\title{
Are dark energy models with variable EoS parameter $w$ compatible with the late inhomogeneous Universe?
}

Özgür Akarsu ${ }^{1}$, Mariam Bouhmadi-López ${ }^{2,3,4,5}$, Maxim Brilenkov ${ }^{6}$, Ruslan Brilenkov ${ }^{6}$, Maxim Eingorn ${ }^{7}$, and Alexander Zhuk $^{8}$

${ }^{1}$ Department of Physics, Koç University, 34450 Sarıer, İstanbul, Turkey

${ }^{2}$ Departamento de Física, Universidade da Beira Interior, 6200 Covilhã, Portugal

${ }^{3}$ Centro de Matemática e Aplicações da Universidade da Beira Interior (CMA-UBI), 6200 Covilhã, Portugal

${ }^{4}$ Department of Theoretical Physics, University of the Basque Country UPV/EHU, P.O. Box 644, 48080 Bilbao, Spain

${ }^{5}$ IKERBASQUE, Basque Foundation for Science, 48011 Bilbao, Spain

${ }^{6}$ Department of Theoretical Physics, Odessa National University,

Street Dvoryanskaya 2, Odessa 65082, Ukraine

${ }^{7}$ North Carolina Central University, CREST and NASA Research Centers, Fayetteville st. 1801, Durham, North Carolina 27707, U.S.A.

${ }^{8}$ Astronomical Observatory, Odessa National University,

Street Dvoryanskaya 2, Odessa 65082, Ukraine 
E-mail: oakarsu@ku.edu.tr, mbl@ubi.pt On leave of absence from UPV and IKERBASQUE, maxim.brilenkov@gmail.com, ruslan.brilenkov@gmail.com, maxim.eingorn@gmail.com, ai.zhuk2@gmail.com

Abstract. We study the late-time evolution of the Universe where dark energy (DE) is presented by a barotropic fluid on top of cold dark matter (CDM). We also take into account the radiation content of the Universe. Here by the late stage of the evolution we refer to the epoch where CDM is already clustered into inhomogeneously distributed discrete structures (galaxies, groups and clusters of galaxies). Under this condition the mechanical approach is an adequate tool to study the Universe deep inside the cell of uniformity. More precisely, we study scalar perturbations of the FLRW metric due to inhomogeneities of CDM as well as fluctuations of radiation and DE. For an arbitrary equation of state for DE we obtain a system of equations for the scalar perturbations within the mechanical approach. First, in the case of a constant DE equation of state parameter $w$, we demonstrate that our method singles out the cosmological constant as the only viable dark energy candidate. Then, we apply our approach to variable equation of state parameters in the form of three different linear parametrizations of $w$, e.g., the Chevallier-Polarski-Linder perfect fluid model. We conclude that all these models are incompatible with the theory of scalar perturbations in the late Universe. 


\section{Contents}

1 Introduction $\quad 1$

2 Basic equations $r$

2.1 Background 2

$\begin{array}{lll}2.2 & \text { Scalar perturbations } & 3\end{array}$

3 CPL model $r$

4 Dark energy with an EoS parameter linear in $z \quad 8$

5 Dark energy with an EoS parameter linear in $t \quad 8$

6 Conclusions

\section{Introduction}

Explaining the accelerated expansion of the late Universe is one of the greatest challenges in modern cosmology. The $\Lambda \mathrm{CDM}$ model is successfully described by the Planck data [1]. However, the nature of the cosmological constant is still unclear. In fact, there are about 120 orders of magnitude between the observed value of the cosmological constant and the theoretically expected value. The latter is related to the vacuum energy density. The cancellation mechanism that would reduce this large theoretical value of the cosmological constant is still a mystery [2]. In addition, the $\Lambda$ CDM model (as well as a lot of other dark energy models) faces the coincidence problem, i.e. why is the cosmological constant at present of the same order of magnitude as the dark matter energy density? To solve these problems, different dynamical dark energy models were proposed. For example, such dark energy can be modeled by a barotropic perfect fluid (i.e. a fluid whose density is a function of its pressure only) with a proper equation of state (EoS). The Chevallier-Polarski-Linder (CPL) parametrization of the EoS $[3,4]$ is often used in literature (see, e.g., the recent papers [5-7]). In this approach the parameter of the EoS is a simple linear function with respect to the scale factor $a$ of the Universe: $w(a)=w_{0}+w_{1}\left(1-a / a_{0}\right)$, where $w_{0,1}$ are free parameters of the model and $a_{0}$ is the present day value of the scale factor. The energy density $\varepsilon_{\mathrm{CPL}}$ of this fluid behaves as $\varepsilon_{\mathrm{CPL}} \sim a^{-3\left(1+w_{0}+w_{1}\right)}$ at early times when $a \ll a_{0}$, and when $a$ is close to $a_{0}$ as well. Therefore, if $w_{0,1}$ are close to -1 and 0 respectively, then this barotropic fluid mimics the cosmological constant. Recently BOSS collaboration gave the constraints on the parameters of this EoS from the Planck+BAO+SN data as $w_{0}=-0.93 \pm 0.11(1 \sigma)$ and $w_{1}=-0.2 \pm 0.4$ $(1 \sigma)$ [8]. In [9] an alternative form of the EoS was suggested following from the linear time parametrization $w(t)=w_{0}+w_{1}\left(1-t / t_{0}\right)$. Using the same data and method with [8], the authors found that the Planck $+\mathrm{BAO}+\mathrm{SN}$ data predict for this model $w_{0}=-0.99 \pm 0.06(1 \sigma)$ and $-1<w_{0}+w_{1}<-0.42(2 \sigma)$.

It is of interest to investigate these models from the point of their compatibility with other cosmological and astrophysical data, as well as with the theory of cosmological perturbations, in particular, with the theory of scalar perturbations in the late Universe. At the late stage of the Universe evolution when inhomogeneities (such as galaxies and their 
groups) are already formed, the hydrodynamic approach is inadequate. Here the mechanical approach $[10,11]$ is more appropriate. It works well inside the cell of uniformity [12] and provides us a good tool to investigate scalar perturbations for different cosmological models (see, e.g., [13-15]). Therefore, it makes sense to study cosmological models filled with perfect fluids which have linear EoS parametrizations and investigate the compatibility of these models with the mechanical approach. Obviously, the above-mentioned forms of the EoS are among the simplest ones. In our paper, for completeness, we also consider a linear parametrization with respect to the redshift [16]: $w(z)=w_{0}-w_{1} z$. As a result, we show that all three considered models are incompatible with the theory of scalar perturbations in the late Universe. This means that such parametrizations can be used only for a limited period of time, starting, e.g., from last scattering till present. That is they follow from some more general EoS and for late times (in the future) they should be replaced by other expressions.

The paper is structured as follows. In Sec. 2 we derive the basic equations for scalar perturbations in the framework of the mechanical approach in the case of a barotropic perfect fluid with an arbitrary EoS $p_{\mathrm{X}}=p_{\mathrm{X}}\left(\varepsilon_{\mathrm{X}}\right)$. Then, in Secs. 3, 4 and 5 we demonstrate that the enumerated DE EoS parametrizations linear in $a, z$ and $t$, respectively, are incompatible with the scalar perturbations theory. The main results are briefly summarized in concluding Sec. 6. In Appendix we consider a particular case of a barotropic perfect fluid with a constant EoS parameter. Here we investigate the conservation equations for scalar perturbations in the mechanical approach and demonstrate its self-consistency.

\section{Basic equations}

\subsection{Background}

We consider the Universe at late stages of its evolution when galaxies and clusters of galaxies have already formed. At scales of the order of approximately $190 \mathrm{Mpc}$ [12], being much larger than the characteristic distance between these inhomogeneities, the Universe is well described by the homogeneous and isotropic FLRW metric and the Friedmann equations

$$
\frac{3\left(\mathcal{H}^{2}+\mathcal{K}\right)}{a^{2}}=\kappa\left(\bar{T}_{0(\mathrm{CDM})}^{0}+\bar{\varepsilon}_{\mathrm{rad}}+\bar{\varepsilon}_{\mathrm{X}}\right)+\Lambda
$$

and

$$
\frac{2 \mathcal{H}^{\prime}+\mathcal{H}^{2}+\mathcal{K}}{a^{2}}=-\kappa\left(\bar{p}_{\text {rad }}+\bar{p}_{\mathrm{X}}\right)+\Lambda
$$

where $\mathcal{H} \equiv a^{\prime} / a \equiv(d a / d \eta) / a, \quad \kappa \equiv 8 \pi G_{N} / c^{4}$ ( $c$ is the speed of light and $G_{N}$ is the Newtonian gravitational constant) and $\mathcal{K}=-1,0,+1$ stands for open, flat and closed Universes, respectively. Conformal time $\eta$ and synchronous time $t$ are connected as $c d t=a d \eta$. Further, $\bar{T}_{k(\mathrm{CDM})}^{i}$ is the average energy-momentum tensor of the pressureless (dust-like) matter. For such matter the energy density $\bar{T}_{0(\mathrm{CDM})}^{0}=\bar{\rho}_{\mathrm{c}} c^{2} / a^{3}$ is the only nonzero component, here $\bar{\rho}_{\mathrm{c}}=$ const is the average comoving rest mass density [10]. As usual, for radiation we have the EoS $\bar{p}_{\text {rad }}=(1 / 3) \bar{\varepsilon}_{\text {rad }}$. The Universe is also supposed to be filled with a barotropic perfect fluid with the EoS $\bar{p}_{\mathrm{X}}=\bar{p}_{\mathrm{X}}\left(\bar{\varepsilon}_{\mathrm{X}}\right)$ corresponding to DE. For generality, we also included the cosmological constant $\Lambda$ into the above equations. Obviously, if the barotropic fluid is the reason of the late time acceleration of the Universe, then the cosmological constant is not necessary. It can be easily seen that the presence (or absence) of the cosmological constant does not affect the scalar perturbation analysis. For example, one can write down the Einstein equations with an upper index and a lower one. Then, the $\Lambda$ term will correspond to 
the contribution $\Lambda \delta_{k}^{i}$ whose perturbations are zero at any order ${ }^{1}$. However, the dynamical behavior of the Universe (i.e. the form of the scale factor $a$ ) depends on $\Lambda$.

From Eqs. (2.1) and (2.2) we can easily get the following auxiliary equation:

$$
\frac{2}{a^{2}}\left(\mathcal{H}^{\prime}-\mathcal{H}^{2}-\mathcal{K}\right)=-\kappa\left(\bar{T}_{0(\mathrm{CDM})}^{0}+\bar{\varepsilon}_{\mathrm{rad}}+\bar{\varepsilon}_{\mathrm{X}}+\bar{p}_{\mathrm{rad}}+\bar{p}_{\mathrm{X}}\right) .
$$

\subsection{Scalar perturbations}

Deep inside the cell of uniformity the Universe is highly inhomogeneous. Here the mechanical approach is more adequate than the hydrodynamical one $[10,12]$. In the framework of the mechanical approach galaxies, dwarf galaxies and clusters of galaxies (composed of baryonic and dark matter) are considered as separate compact objects. Moreover, at distances much greater than their characteristic sizes they can be described well as point-like matter sources. This is a generalization of the well-known astrophysical approach [18] (see §106) for the case of the dynamical cosmological background. Usually, the gravitational fields of galaxies are weak and their peculiar velocities are much smaller than the speed of light. These inhomogeneities together with fluctuations of other matter sources result in scalar perturbations of the FLRW metric $[19,20]$. In the mechanical approach and in the conformal Newtonian gauge, the gravitational potential (i.e. the perturbation of the metric coefficient $\left.g_{00}\right) \Phi \sim O\left(1 / c^{2}\right)$ satisfies the following system of equations (see $[10,12,14]$ for details):

$$
\begin{aligned}
& \Delta \Phi-3 \mathcal{H}\left(\Phi^{\prime}+\mathcal{H} \Phi\right)+3 \mathcal{K} \Phi=\frac{1}{2} \kappa a^{2}\left(\delta T_{0(\mathrm{CDM})}^{0}+\delta \varepsilon_{\mathrm{X}}+\delta \varepsilon_{\mathrm{rad} 1}+\delta \varepsilon_{\mathrm{rad} 2}\right), \\
& \frac{\partial}{\partial x^{\beta}}\left(\Phi^{\prime}+\mathcal{H} \Phi\right)=0, \\
& \Phi^{\prime \prime}+3 \mathcal{H} \Phi^{\prime}+\left(2 \mathcal{H}^{\prime}+\mathcal{H}^{2}\right) \Phi-\mathcal{K} \Phi=\frac{1}{2} \kappa a^{2}\left(\delta p_{\mathrm{X}}+\delta p_{\mathrm{rad} 1}+\delta p_{\mathrm{rad} 2}\right),
\end{aligned}
$$

where the Laplace operator $\triangle$ is defined with respect to the conformal spatial metric, and $x^{\beta}, \beta=1,2,3$, are the comoving spatial coordinates. From Eq. (2.5) we get immediately that

$$
\Phi(\eta, \mathbf{r})=\frac{\varphi(\mathbf{r})}{c^{2} a(\eta)},
$$

where $\varphi(\mathbf{r})$ is a function of all comoving spatial coordinates, and we have introduced $c^{2}$ in the denominator for convenience. In the surrounding of an inhomogeneity, the comoving potential $\varphi(\mathbf{r}) \sim 1 / r[10,12,13]$, and the nonrelativistic gravitational potential $\Phi(\eta, \mathbf{r}) \sim$ $1 /(\operatorname{ar})=1 / R$, where $R=a r$ is the physical distance. Hence, $\Phi$ has the correct Newtonian limit near the inhomogeneities.

Concerning the fluctuations of the matter sources, $\delta T_{0}^{0}(\mathrm{CDM})$ is related to the fluctuation of the energy density of dust-like matter and has the form ${ }^{2}[10]$

$$
\delta T_{0(\mathrm{CDM})}^{0}=\frac{\delta \rho_{\mathrm{c}} c^{2}}{a^{3}}+\frac{3 \bar{\rho}_{\mathrm{c}} c^{2} \Phi}{a^{3}},
$$

\footnotetext{
${ }^{1}$ The conservation equation clearly shows that the $\Lambda$ term can vary only in the case of interaction with other matter (see, e.g., [17]). However, this is out of the scope of our model.

${ }^{2}$ It can be easily seen from Eq. (2.23) in [10] that these fluctuations read $\delta T_{0(\mathrm{CDM})}^{0}=\delta \rho_{\mathrm{c}} c^{2} / a^{3}+$ $\left(\bar{\rho}_{\mathrm{c}} c^{2} / a^{3}\right)\left[3 \Phi+\alpha \Phi^{2}+\beta \Phi^{3}+\ldots\right]$ where $\alpha$ and $\beta$ are some constants. Taking into account Eq. (2.7), we get $\Phi / a^{3} \sim O\left(1 / a^{4}\right), \Phi^{2} / a^{3} \sim O\left(1 / a^{5}\right), \Phi^{3} / a^{3} \sim O\left(1 / a^{6}\right)$ and so on. Since we consider linear perturbations, we should drop the terms $\Phi^{2}, \Phi^{3}, \ldots$ Therefore, in what follows, we will keep terms up to the order $O\left(1 / a^{4}\right)$ inclusive.
} 
where $\delta \rho_{\mathrm{c}}$ is the difference between the real and average comoving rest mass densities: $\delta \rho_{\mathrm{c}}=\rho_{\mathrm{c}}-\bar{\rho}_{\mathrm{c}}$. We also split the fluctuations of radiation into two parts. The part labeled by "rad1" is caused by the inhomogeneities of dust-like matter (e.g., by galaxies and their groups), and the part labeled by "rad2" is related to fluctuations of the additional perfect fluid (labeled by $X$ ). Of course, in the experiments we measure the total fluctuation of radiation. However, inhomogeneities associated with different matter sources can contribute to this total fluctuation separately. With respect to the additional perfect fluid, as we will see below, such contributions may take place directly via nonzero $\delta \varepsilon_{\operatorname{rad} 2}$ (cf. Eq. (2.22)). This happens if the inhomogeneities of the additional perfect fluid are distributed independently of the fluctuations of CDM [14]. However, there is also the possibility that the fluctuations of this perfect fluid nest on the inhomogeneities of CDM (such as galaxies, groups of galaxies, etc) screening the latter. In such models, $\delta \varepsilon_{\operatorname{rad} 2}=0, \delta \varepsilon_{\mathrm{X}} \sim \varphi$ and Eq. (2.17) (see below) is reduced (for physically consistent cases) to the Helmholtz equation [13]. Then, the fluctuations of the additional perfect fluid contribute to the fluctuations of radiation indirectly via the comoving gravitational potential $\varphi(\mathbf{r})$ (cf. Eq. (2.10)) which is a solution of the corresponding Helmholtz equation. Given these two possible scenarios, we include $\delta \varepsilon_{\operatorname{rad} 2}$ in our equations. Only a detailed analysis of a given model reveals which of these two scenarios is realized.

For both of these contributions we have the same equations of state:

$$
\delta p_{\mathrm{rad} 1}=(1 / 3) \delta \varepsilon_{\mathrm{rad} 1}, \quad \delta p_{\mathrm{rad} 2}=(1 / 3) \delta \varepsilon_{\mathrm{rad} 2} .
$$

We have shown in [12] that $\delta \varepsilon_{\text {rad1 }}$ has the form

$$
\delta \varepsilon_{\mathrm{rad} 1}=-\frac{3 \bar{\rho}_{\mathrm{c}} \varphi}{a^{4}} .
$$

It is worth noting that $\delta \varepsilon_{\text {rad2 }}$ should also behave as $1 / a^{4}$ (see Appendix).

Let us now analyze Eqs. (2.4) and (2.6). Taking into account Eq. (2.7), we can rewrite them as follows:

$$
\begin{aligned}
& \Delta \Phi+3 \mathcal{K} \Phi=\frac{1}{2} \kappa a^{2}\left(\delta T_{0(\mathrm{CDM})}^{0}+\delta \varepsilon_{\mathrm{X}}+\delta \varepsilon_{\mathrm{rad} 1}+\delta \varepsilon_{\mathrm{rad} 2}\right) \\
& \left(\mathcal{H}^{\prime}-\mathcal{H}^{2}-\mathcal{K}\right) \Phi=\frac{1}{2} \kappa a^{2}\left(\delta p_{\mathrm{X}}+\delta p_{\mathrm{rad} 1}+\delta p_{\mathrm{rad} 2}\right) .
\end{aligned}
$$

Substituting Eqs. (2.8) and (2.10) into the right hand side (rhs) of Eq. (2.11), we obtain

$$
\Delta \Phi+3 \mathcal{K} \Phi=\frac{1}{2} \kappa a^{2}\left(\frac{\delta \rho_{\mathrm{c}} c^{2}}{a^{3}}+\delta \varepsilon_{\mathrm{X}}+\delta \varepsilon_{\mathrm{rad} 2}\right) .
$$

On the other hand, substituting (2.3) into the left hand side (lhs) of Eq. (2.12), we get the equation

$$
-\left(\frac{\bar{\rho}_{\mathrm{c}} c^{2}}{a^{3}}+\bar{\varepsilon}_{\mathrm{rad}}+\bar{\varepsilon}_{\mathrm{X}}+\bar{p}_{\mathrm{rad}}+\bar{p}_{\mathrm{X}}\right) \Phi=\delta p_{\mathrm{X}}+\delta p_{\mathrm{rad} 1}+\delta p_{\mathrm{rad} 2} .
$$

Now, for consistency, we must take into account terms up to the order $O\left(1 / a^{4}\right)$ inclusive. This is the accuracy of our investigations. Then, the terms $\bar{\varepsilon}_{\text {rad }} \Phi$ and $\bar{p}_{\text {rad }} \Phi$ of the order $O\left(1 / a^{5}\right)($ cf. Eq. (2.7)) should be dropped:

$$
-\left(\frac{\bar{\rho}_{\mathrm{c}} c^{2}}{a^{3}}+\bar{\varepsilon}_{\mathrm{X}}+\bar{p}_{\mathrm{X}}\right) \Phi=\delta p_{\mathrm{X}}+\delta p_{\mathrm{rad} 1}+\delta p_{\mathrm{rad} 2} .
$$


This equation can be rewritten in the form

$$
-\left(\bar{\varepsilon}_{\mathrm{X}}+\bar{p}_{\mathrm{X}}\right) \Phi=\delta p_{\mathrm{X}}+\frac{1}{3} \delta \varepsilon_{\mathrm{rad} 2},
$$

where we have used Eqs. (2.9) and (2.10).

Therefore, Eqs. (2.4) and (2.6) are reduced to the following system:

$$
\begin{aligned}
& \triangle \varphi+3 \mathcal{K} \varphi=\frac{\kappa c^{4}}{2} \delta \rho_{\mathrm{c}}+\frac{\kappa c^{2} a^{3}}{2} \delta \varepsilon_{\mathrm{X}}+\frac{\kappa c^{2} a^{3}}{2} \delta \varepsilon_{\mathrm{rad} 2}, \\
& -\left(\bar{\varepsilon}_{\mathrm{X}}+\bar{p}_{\mathrm{X}}\right) \frac{\varphi}{c^{2} a}=\delta p_{\mathrm{X}}+\frac{1}{3} \delta \varepsilon_{\mathrm{rad} 2} .
\end{aligned}
$$

Obviously, DE described by the barotropic perfect fluid must satisfy this system at any moment of the cosmic time in the late Universe. Such analysis should be performed for each EoS $\bar{p}_{\mathrm{X}}=\bar{p}_{\mathrm{X}}\left(\bar{\varepsilon}_{\mathrm{X}}\right)$ individually.

For example, we can consider a perfect fluid with a constant parameter in the linear EoS:

$$
\bar{p}_{\mathrm{X}}=w \bar{\varepsilon}_{\mathrm{X}}, \quad w \neq 0 .
$$

We exclude the case $w=0$ since the CDM component was already taken into account in Eq. $(2.1)^{3}$. From the conservation equation we have

$$
\bar{\varepsilon}_{\mathrm{X}}=\frac{\bar{A}}{a^{3(1+w)}} .
$$

Further, for fluctuations of this matter we have

$$
\delta p_{\mathrm{X}}=w \delta \varepsilon_{\mathrm{X}} .
$$

Therefore, Eq. (2.18) gives

$$
\delta \varepsilon_{\mathrm{X}}=-\frac{\varphi}{c^{2} a} \frac{1+w}{w} \bar{\varepsilon}_{\mathrm{X}}-\frac{1}{3 w} \delta \varepsilon_{\mathrm{rad} 2} .
$$

Substituting this relation into (2.17), we arrive at the following equation:

$$
\triangle \varphi+3 \mathcal{K} \varphi-\frac{\kappa c^{4}}{2} \delta \rho_{\mathrm{c}}=-\frac{\kappa \varphi}{2} \frac{1+w}{w} a^{2} \bar{\varepsilon}_{\mathrm{X}}+\frac{\kappa c^{2} a^{3}}{2}\left(1-\frac{1}{3 w}\right) \delta \varepsilon_{\mathrm{rad} 2} .
$$

The lhs of this equation does not depend on time ${ }^{4}$. Therefore, the same should hold true for its rhs. Because $\delta \varepsilon_{\mathrm{rad} 2} \sim 1 / a^{4}$, we must demand that $\delta \varepsilon_{\mathrm{rad} 2} \equiv 0$ (there is no possibility that the term with $\bar{\varepsilon}_{\mathrm{X}}$ and the term with $\delta \varepsilon_{\mathrm{rad} 2}$ cancel each other). Therefore, there are only two values of $w$ which do not contradict this equation. They are $w=-1$ and $w=-1 / 3$. The former case reduces to the standard $\Lambda \mathrm{CDM}$ model considered in [10]. The latter case $w=-1 / 3$ was considered in detail in [13] in the absence of radiation. The same conclusions

\footnotetext{
${ }^{3}$ In addition, if $w=0$ : (i) we get from Eq. (2.18) that $\delta \varepsilon_{\operatorname{rad} 2}=-\bar{\varepsilon}_{\mathbf{X}}\left[3 \varphi /\left(c^{2} a\right)\right]=-3 \bar{A} \varphi /\left(c^{2} a^{4}\right)$ which is similar to Eq. (2.10), and (ii) Eq. (2.17) implies $\delta \varepsilon_{\mathrm{X}}=B / a^{3}-\delta \varepsilon_{\mathrm{rad} 2}$ in full analogy with Eq. (2.8) (for $\left.B \equiv \delta \rho_{\mathrm{cX}} c^{2}\right)$. Obviously, we can combine both of these dust components into a single one. Therefore, we would be back to the model with CDM and a $\Lambda$ term (in the background equations (2.1) and (2.2)).

${ }^{4} \mathrm{We}$ would like to remind that the quantities $\varphi$ and $\delta \rho_{\mathrm{c}}$ are the comoving ones [10]. Therefore, within the adopted accuracy when both nonrelativistic and weak field limits are applied, they do not depend explicitly on time [12].
} 
with respect to the model with constant $w$ can be obtained from the conservation equations (see Appendix). Obviously, the dark energy component is smooth and homogeneous in the background equations where matter/energy is considered in the averaged form. However, it does not have to be smooth and homogeneous a priori. The conclusion of the presence or absence of dark energy fluctuations follows from the analysis of Einstein equations for perturbations. For example, for the $\Lambda \mathrm{CDM}$ model (i.e. $w=-1$ ) fluctuations are absent, as it is easily seen from Eq. (2.22) where $\delta \varepsilon_{\mathrm{rad} 2}=0$ (see also [10]), so dark energy represented by the cosmological constant $\Lambda$ is truly homogeneous, while for the model with $w=-1 / 3$, fluctuations are nonzero [13], so there are inhomogeneities in the dark energy distribution over the Universe. It is clear that in the case of the inhomogeneous distribution, the average value of fluctuations should be equal to zero: $\overline{\delta \varepsilon_{\mathrm{X}}}=0$ (see, e.g., [13] where it is shown explicitly).

We next investigate the models with dynamical $w$.

\section{CPL model}

First, we consider the CPL model (i.e. $\mathrm{X} \equiv \mathrm{CPL}$ ) and the corresponding EoS $[3,4]$ :

$$
\bar{p}_{\mathrm{CPL}}=w(a) \bar{\varepsilon}_{\mathrm{CPL}}, \quad w(a)=w_{0}+w_{1}\left(1-\frac{a}{a_{0}}\right),
$$

where $a_{0}$ represents the scale factor of the Universe at the present time. We consider this EoS to be valid for a definite period of time in the past (e.g., from last scattering [4]). We also suppose that this EoS is still valid for some period of time in the future.

From the conservation equation in the form

$$
d\left(\bar{\varepsilon}_{\mathrm{CPL}} a^{3}\right)+\bar{p}_{\mathrm{CPL}} d\left(a^{3}\right)=0
$$

we easily get the well known formula

$$
\bar{\varepsilon}_{\mathrm{CPL}}=A a^{-3\left(1+w_{0}+w_{1}\right)} e^{3 w_{1} a / a_{0}},
$$

where $A$ is a constant of integration. Therefore,

$$
\bar{p}_{\mathrm{CPL}}+\bar{\varepsilon}_{\mathrm{CPL}}=A a^{-3\left(1+w_{0}+w_{1}\right)} e^{3 w_{1} a / a_{0}}\left[1+w_{0}+w_{1}\left(1-\frac{a}{a_{0}}\right)\right] .
$$

Additionally, with the help of Eq. (3.3), we obtain the expression for the fluctuation of the CPL fluid pressure:

$$
\begin{aligned}
\delta p_{\mathrm{CPL}} & =\frac{\partial\left(w \bar{\varepsilon}_{\mathrm{CPL}}\right)}{\partial \bar{\varepsilon}_{\mathrm{CPL}}} \delta \varepsilon_{\mathrm{CPL}}=\left(w-\frac{\left.w_{1} \bar{\varepsilon}_{\mathrm{CPL}} \frac{d a}{a_{0}}\right) \delta \varepsilon_{\mathrm{CPL}}}{d \bar{\varepsilon}_{\mathrm{CPL}}}\right) \\
& =\left[w_{0}+w_{1}\left(1-\frac{a}{a_{0}}\right)-\frac{1}{3} \frac{w_{1}}{w_{1}-\frac{a_{0}}{a}\left(1+w_{0}+w_{1}\right)}\right] \delta \varepsilon_{\mathrm{CPL}} .
\end{aligned}
$$

Therefore, Eq. (2.18) reads

$$
\begin{array}{r}
-\frac{A \varphi}{c^{2}} a^{-3\left(1+w_{0}+w_{1}\right)-1} e^{3 w_{1} a / a_{0}}\left[1+w_{0}+w_{1}\left(1-\frac{a}{a_{0}}\right)\right] \\
=\left[w_{0}+w_{1}\left(1-\frac{a}{a_{0}}\right)-\frac{1}{3} \frac{w_{1}}{w_{1}-\frac{a_{0}}{a}\left(1+w_{0}+w_{1}\right)}\right] \delta \varepsilon_{\mathrm{CPL}}+\frac{1}{3} \delta \varepsilon_{\mathrm{rad} 2},
\end{array}
$$


and for $\delta \varepsilon_{\mathrm{CPL}}$ we have

$$
\begin{aligned}
\delta \varepsilon_{\mathrm{CPL}} & =-\frac{A \varphi}{c^{2}} a^{-3\left(1+w_{0}+w_{1}\right)-1} e^{3 w_{1} \beta} \frac{\left(1+w_{0}+w_{1}(1-\beta)\right)^{2}}{w_{0}+w_{1}(1-2 \beta / 3)+\left(w_{0}+w_{1}(1-\beta)\right)^{2}} \\
& -\frac{1}{3} \delta \varepsilon_{\operatorname{rad} 2} \frac{1+w_{0}+w_{1}(1-\beta)}{w_{0}+w_{1}(1-2 \beta / 3)+\left(w_{0}+w_{1}(1-\beta)\right)^{2}},
\end{aligned}
$$

where for convenience we have introduced the notation $\beta \equiv a / a_{0}$. Consequently, Eq. (2.17) takes the form

$$
\begin{aligned}
\triangle \varphi+3 \mathcal{K} \varphi & =\frac{\kappa c^{4}}{2} \delta \rho_{\mathrm{c}}-\frac{A \kappa \varphi}{2} a^{-3\left(1+w_{0}+w_{1}\right)+2} e^{3 w_{1} \beta} \frac{\left(1+w_{0}+w_{1}(1-\beta)\right)^{2}}{w_{0}+w_{1}(1-2 \beta / 3)+\left(w_{0}+w_{1}(1-\beta)\right)^{2}} \\
+ & \frac{\kappa c^{2} a^{3}}{2} \delta \varepsilon_{\operatorname{rad} 2}\left[1-\frac{1}{3} \frac{1+w_{0}+w_{1}(1-\beta)}{w_{0}+w_{1}(1-2 \beta / 3)+\left(w_{0}+w_{1}(1-\beta)\right)^{2}}\right] .
\end{aligned}
$$

The particular case $w_{1}=0$, i.e. $w=w_{0}=$ const, was considered at the very end of the previous section. Now, we turn to the general case $w_{1} \neq 0$ and consider Eq. (3.8). Here the lhs and the term containing $\delta \rho_{\mathrm{c}}$ are independent of time. Therefore, either each of two remaining expressions are also independent of time (within our accuracy $O(1 / a)$, bearing in mind that the rhs of (3.8) has been multiplied by $a^{3}$ ), or they must cancel each other at any arbitrary moment of time. Can this be the case? The second assumption that the second and third terms on the rhs of Eq. (3.8) can cancel each other at any arbitrary moment of time does not work because of the presence of the exponential (with respect to the scale factor $a$ ) function $\exp \left(3 w_{1} \beta\right)$ which is linearly independent from the power functions. It can be easily seen that the first assumption does not work as well in the case $w_{1} \neq 0$. For example, we can eliminate the last term in Eq. (3.8) by putting $\delta \varepsilon_{\mathrm{rad} 2} \equiv 0$. However, the second term on the rhs of this equation still depends on time. The simple analysis shows that, in contrast to Eq. (2.23), we cannot select the parameters $w_{0,1}$ of the model in such a way that this term becomes independent of time. Already here, we can see a potential problem of the model. However, this term can still be of the order $o(1 / a)$ and then we can drop it within the accuracy of our approach. The problem is that we cannot expand the exponential prefactor into series with respect to the scale factor which can be arbitrary large. Therefore, we need to estimate the second term taking into account the exponential function. Obviously, this term is exponentially small for $w_{1}<0$ and $\beta \gg 1^{5}$. However, we cannot drop the second term simply on the basis of this argument. To show it, we consider the following ratio:

$$
\begin{aligned}
& -\frac{A \kappa \varphi}{2} a^{-3\left(1+w_{0}+w_{1}\right)+2} e^{3 w_{1} \beta} \frac{1}{\frac{\kappa c^{2} a^{3}}{2} \delta \varepsilon_{\mathrm{rad} 1}} \\
& =-\frac{A \kappa \varphi}{2} a^{-3\left(1+w_{0}+w_{1}\right)+2} e^{3 w_{1} \beta} \frac{1}{-\frac{\kappa c^{2} a^{3}}{2} \frac{3 \overline{\rho_{\mathrm{c}}} \varphi}{a^{4}}}=\frac{\bar{\varepsilon}_{\mathrm{CPL}}}{3 \bar{\rho}_{\mathrm{c}} c^{2} / a^{3}} .
\end{aligned}
$$

Now, we assume that CPL matter is responsible for the late time acceleration of the Universe. According to the recent observations, it contributes approximately $70 \%$ to the total energy density of the Universe, while CDM + baryons give approximately the remaining $30 \%$. Hence, at the present moment the ratio (3.9) is approximately equal to 0.78 . If we consider for $w_{0}$ and $w_{1}$ the values -0.93 and -0.2 [8], respectively, then at the present moment (i.e.

\footnotetext{
${ }^{5}$ For positive values of $w_{1}>0$, the second term is exponentially divergent when $a \rightarrow \infty$. Hence, there is no need to consider such values since our model must be valid for arbitrary large values of the scale factor $a$.
} 
for $\beta \sim 1$ ) we get that the second term amounts $3 \%$ from the radiation which we take into account in our analysis. This amount is not negligibly small and we cannot neglect such a time dependent term. Therefore, the considered CPL barotropic fluid with $w_{1} \neq 0$ contradicts the theory of scalar perturbations in the late Universe.

\section{Dark energy with an EoS parameter linear in $z$}

Now, we consider a model where the parameter of the EoS for DE is a linear function of the redshift $z[16]$ :

$$
\bar{p}_{z}=w(z) \bar{\varepsilon}_{z}, \quad w(z)=w_{0}-w_{1} z=w_{0}+w_{1}\left(1-\frac{a_{0}}{a}\right) .
$$

For this perfect fluid we get

$$
\bar{\varepsilon}_{z}=A a^{-3\left(1+w_{0}+w_{1}\right)} e^{-3 w_{1} a_{0} / a} .
$$

By performing an analysis similar to the one carried on the previous section, we conclude that this model also contradicts the theory of scalar perturbations. Obviously, it happens because of the exponential factor in Eq. (4.2) which leads to an equation for the comoving gravitational potential similar to (3.8) where the last two terms on the rhs do not cancel each other.

\section{Dark energy with an EoS parameter linear in $t$}

In the two previous parametrizations of the EoS $w$ is a divergent function either for $a \rightarrow+\infty$ or for $a \rightarrow 0$. In the recent work [9] a different parametrization for $w$ which is based on a linear dependence on time $t$ was proposed:

$$
w(t)=w_{0}+w_{1}\left(1-\frac{t}{t_{0}}\right),
$$

leading to the following expression in terms of the scale factor $a$ :

$$
\begin{aligned}
w_{t}(a) & =w_{0}+w_{1}\left[1-\frac{2\left(1+w_{0}+w_{1}\right)}{w_{1}+\left(2+2 w_{0}+w_{1}\right) \beta^{-3\left(1+w_{0}+w_{1}\right) / 2}}\right] \\
& =\frac{-w_{1}\left(w_{0}+w_{1}+2\right) \beta^{3 \bar{\omega} / 2}+\left(2 w_{0}+w_{1}+2\right)\left(w_{0}+w_{1}\right)}{w_{1} \beta^{3 \bar{\omega} / 2}+2 w_{0}+w_{1}+2} .
\end{aligned}
$$

It was shown in [9] that if DE is parametrized by the EoS (5.2) then: (i) data from Planck, $\mathrm{BAO}$ and SN constrain much better the free parameters of this model (i.e. $w_{0}$ and $w_{1}$ ) as compared with the CPL parametrization, and (ii) if $\left(w_{0}, w_{1}\right)=(-1,1 / 3)$ in Eq. (5.2), i.e. the DE EoS parameter varies between $-2 / 3$ in the early Universe and -1 at present, it fits the data slightly better than a pure $\Lambda$ term. The relation (5.2) was obtained under the assumption $\bar{w} \neq 0$, where $\bar{w} \equiv w_{0}+w_{1}+1$. More precisely, $0<w_{1}<2 \bar{w}$. In general, we can analytically continue this expression to negative values of $\bar{w}$, keeping the condition $\bar{w} \neq 0$. As we will see below, physical quantities (e.g., the energy density) have the same asymptotic behavior at big values of the scale factor $a$ for both positive and negative $\bar{w}$. The EoS parameter (5.2) goes to a constant value in the limit $a \rightarrow+\infty$ : $w_{t}(a) \rightarrow-(\bar{w}+1), \bar{w}>0$ and $w_{t}(a) \rightarrow$ $-(|\bar{w}|+1), \bar{w}<0$. In the case $w_{1}=0$ we naturally have $w_{t}(a)=w_{0}=$ const which was 
considered at the end of Sec. 2. We note that the direct substitution of $\bar{w}=0$ into Eq. (5.2) results in an uncertainty of the type $0 / 0$. One can check that $w_{t}(a) \rightarrow \frac{-3\left(w_{0}+1\right) \ln (\beta)-4 w_{0}}{3\left(w_{0}+1\right) \ln (\beta)-4}$ as $w_{0} \rightarrow-1-w_{1}$. This is not a rational fraction and we again will have a problem (except for the trivial case $w_{0}=-1$ ) similar to the one described in two previous sections. Therefore, we disregard this case.

The conservation equation for the considered fluid reads

$$
d\left(\bar{\varepsilon}_{t} \beta^{3}\right)+w_{t} \bar{\varepsilon}_{t} d\left(\beta^{3}\right)=0 .
$$

Now, with the EoS parameter (5.2), we get the energy density

$$
\bar{\varepsilon}_{t}=\bar{\varepsilon}_{t_{0}}\left(1+\frac{w_{1} \beta^{3 \bar{w} / 2}-w_{1}}{2 \bar{w}}\right)^{4} \beta^{-3 \bar{w}}
$$

where $\bar{\varepsilon}_{t_{0}}$ is an integration constant. It can be easily seen that for both positive and negative $\bar{w}$ the energy density behaves asymptotically as $\bar{\varepsilon}_{t} \sim \beta^{3|\bar{w}|} \rightarrow+\infty$ for $\beta \rightarrow+\infty$. In the case $w_{1}=0$ this formula is reduced to the familiar expression: $\bar{\varepsilon}_{t}=\bar{\varepsilon}_{t_{0}} \beta^{-3\left(1+w_{0}\right)}$.

Let us turn now to the scalar perturbations for this model. Taking into account Eq. (5.4), we get

$$
\bar{p}_{t}+\bar{\varepsilon}_{t}=\bar{\varepsilon}_{t}\left(1+w_{t}\right)=\bar{\varepsilon}_{t_{0}} \bar{w} \beta^{-3 \bar{w}}\left(1+\frac{w_{1} \beta^{3 \bar{w} / 2}-w_{1}}{2 \bar{w}}\right)^{3}\left(1-\frac{w_{1} \beta^{3 \bar{w} / 2}+w_{1}}{2 \bar{w}}\right),
$$

and

$$
\begin{aligned}
& \delta p_{t}=\frac{\partial\left(w_{t} \bar{\varepsilon}_{t}\right)}{\partial \bar{\varepsilon}_{t}} \delta \varepsilon_{t}=\left(w_{t}+\frac{\partial w_{t}}{\partial a} \frac{\partial a}{\partial \bar{\varepsilon}_{t}} \bar{\varepsilon}_{t}\right) \delta \varepsilon_{t} \\
= & {\left[\frac{-w_{1}(\bar{w}+1) \beta^{3 \bar{w} / 2}+\left(2 \bar{w}-w_{1}\right)(\bar{w}-1)}{w_{1} \beta^{3 \bar{w} / 2}+2 \bar{w}-w_{1}}+\frac{w_{1}\left(2 \bar{w}-w_{1}\right) \bar{w}}{-w_{1}^{2} \beta^{3 \bar{w} / 2}+\left(2 \bar{w}-w_{1}\right)^{2} \beta^{-3 \bar{w} / 2}}\right] \delta \varepsilon_{t} . }
\end{aligned}
$$

With the help of Eq. (2.18) we obtain the expression for $\delta \varepsilon_{t}$ :

$$
\delta \varepsilon_{t}=\frac{-\frac{\varphi}{c^{2} a_{0}} \bar{\varepsilon}_{t_{0}} \bar{w} \beta^{-3 \bar{w}-1}\left(1+\frac{w_{1} \beta^{3 \bar{w} / 2}-w_{1}}{2 \bar{w}}\right)^{3}\left(1-\frac{w_{1} \beta^{3 \bar{w} / 2}+w_{1}}{2 \bar{w}}\right)-\frac{1}{3} \delta \varepsilon_{\mathrm{rad} 2}}{\frac{-w_{1}(\bar{w}+1) \beta^{3 \bar{w} / 2}+\left(2 \bar{w}-w_{1}\right)(\bar{w}-1)}{w_{1} \beta^{3 \bar{w}} / 2+2 \bar{w}-w_{1}}+\frac{w_{1}\left(2 \bar{w}-w_{1}\right) \bar{w}}{-w_{1}^{2} \beta^{3 \bar{w} / 2}+\left(2 \bar{w}-w_{1}\right)^{2} \beta^{-3 \bar{w} / 2}}} .
$$

Then, Eq. (2.17) takes the form

$$
\begin{aligned}
& \Delta \varphi+3 \mathcal{K} \varphi=\frac{\kappa c^{4}}{2} \delta \rho_{\mathrm{c}} \\
& -\frac{\bar{\varepsilon}_{t_{0}} \kappa \varphi a_{0}^{2}}{2}\left[\frac{\bar{w} \beta^{-3 \bar{w}+2}\left(1+\frac{w_{1} \beta^{3 \bar{w} / 2}-w_{1}}{2 \bar{w}}\right)^{3}\left(1-\frac{w_{1} \beta^{3 \bar{w} / 2}+w_{1}}{2 \bar{w}}\right)}{\frac{-w_{1}(\bar{w}+1) \beta^{3 \bar{w} / 2}+\left(2 \bar{w}-w_{1}\right)(\bar{w}-1)}{w_{1} \beta^{3 \bar{w} / 2}+2 \bar{w}-w_{1}}+\frac{w_{1}\left(2 \bar{w}-w_{1}\right) \bar{w}}{-w_{1}^{2} \beta^{3 \bar{w} / 2}+\left(2 \bar{w}-w_{1}\right)^{2} \beta^{-3 \bar{w} / 2}}}\right] \\
& +\frac{\kappa c^{2} a_{0}^{3} \beta^{3}}{2} \delta \varepsilon_{\operatorname{rad} 2}\left[1-\frac{1}{\frac{-3 w_{1}(\bar{w}+1) \beta^{3 \bar{w} / 2}+3\left(2 \bar{w}-w_{1}\right)(\bar{w}-1)}{w_{1} \beta^{3 \bar{w} / 2}+2 \bar{w}-w_{1}}+\frac{3 w_{1}\left(2 \bar{w}-w_{1}\right) \bar{w}}{-w_{1}^{2} \beta^{3 \bar{w}} / 2+\left(2 \bar{w}-w_{1}\right)^{2} \beta^{-3 \bar{w} / 2}}}\right]
\end{aligned}
$$

It can be easily seen that this equation reduces to Eq. (2.23) for the particular value $w_{1}=0$ and, consequently, $w=w_{0}$. Let us now consider the general case $w_{1} \neq 0$. According to 
the accuracy of our approach, we should take into account all terms up to the order $O(1 / a)$ inclusive (bearing in mind that the rhs of (5.8) has been multiplied by $a^{3}$ ). It should take place for all times in the late Universe including the far future $\beta \gg 1$. In this limit it is very convenient to expand the expressions under consideration in powers of the scale factor. We see that, because the first line in Eq. (5.8) is independent of time (or of the scale factor), all terms of the order $O\left(1 / a^{n}\right), n \leq 1$ in the next lines must be absent or they must cancel each other. The term in the second line behaves asymptotically as $\sim \beta^{3|\bar{w}|+2}$, when $\beta \gg 1$, i.e. its asymptotic behavior does not depend on the sign of $\bar{w}$. The last term behaves asymptotically as $\sim \beta^{3} \delta \varepsilon_{\operatorname{rad} 2}$, if $\beta \gg 1$, again for both positive and negative $\bar{w}$. Therefore, this term has the asymptotic form $\sim 1 / \beta$ in the presence of $\delta \varepsilon_{\operatorname{rad} 2} \sim 1 / a^{4}$ or it equals identically to zero for $\delta \varepsilon_{\mathrm{rad} 2} \equiv 0$. Hence, first, in the presence of $\delta \varepsilon_{\mathrm{rad} 2}$ the second line and the last term cannot cancel each other, and, second, in the absence of such radiation the time dependent second line cannot be dropped from the equation. This result is in contradiction. We conclude that the problem has arisen because of the power-law growth of the energy density in the expanding Universe: $\bar{\varepsilon}_{t} \sim \beta^{3}|\bar{w}|$ when $\beta \gg 1$. Therefore, the model considered in this section contradicts as well the theory of scalar perturbations in the late Universe.

\section{Conclusions}

In our paper we have studied the late Universe filled with dust-like matter and a barotropic perfect fluid which can play the role of dark energy. We have considered the radiation content of the Universe as well. More precisely, we have concentrated on scales much smaller than the cell of uniformity size which is approximately $190 \mathrm{Mpc}$ [12]. At such distances our Universe is highly inhomogeneous and the averaged Friedmann approach does not work here because we need to take into account inhomogeneities in the form of galaxies, groups and clusters of galaxies. It is also a natural assumption that radiation and the barotropic perfect fluid fluctuate around the average values. Therefore, these fluctuations as well as the dust-like matter inhomogeneities perturb the FLRW metric. To analyze these perturbations inside the cell of uniformity, we need to use the mechanical approach [10-12]. An important feature of this approach is that it provides an optimal tool to study the compatibility of different cosmological models with observations (see, e.g., [13, 14]).

In the present paper we have firstly derived the equations for scalar perturbations within the mechanical approach in the case of a barotropic fluid with an arbitrary EoS $p_{\mathrm{X}}=p_{\mathrm{X}}\left(\varepsilon_{\mathrm{X}}\right)$ (on top of the dust-like matter and radiation content of the Universe). Our master equations are Eqs. (2.17) and (2.18). First, we have shown how these equations work in the simplest case of a constant EoS parameter $w$ for dark energy. Our analysis demonstrates that the only two values of $w$, namely $w=-1$ and $w=-1 / 3$, are acceptable. Therefore, in the case of constant $w$, the cosmological constant (i.e. $w=-1$ ) is the only viable dark energy candidate (since a perfect fluid with $w=-1 / 3$ does not accelerate the Universe).

Then we have applied these equations to variable EoS parameters. Since it is impossible to handle this problem in full generality, we consider three linear parametrizations of $w$ : CPL (i.e., the parameter of the EoS is linear in the scale factor $a$ ), linear in the redshift $z$ and linear in time $t$. Our analysis has shown that all these three models contradict the theory of scalar perturbations in the late Universe. This occurs despite the fact that these models can be in sufficient agreement with the observed data obtained from Planck, BAO and SN $[1,9,16]$. This means that these EoS are not the fundamental ones and they can be used only for a limited period of time, starting, e.g., from the time of last scattering and up to the present 
moment. During this period they approximate/mimic more or less successfully the unknown, "real" EoS for DE. In the future Universe they should be replaced by other expressions. Our investigations show that the most simple and usual constituents of the Universe such as the cosmological constant, pressureless dark and baryonic matter and radiation are in full agreement with the theory of scalar perturbations. Interestingly, a perfect fluid with a constant EoS parameter $w=-1 / 3$ fits this scheme also very well [13].

We highlight that our conclusion on the incompatibility of perfect fluids which have variable EoS parameters with the theory of scalar perturbations in the late Universe applies only to the considered cases of linear parametrizations of $w$. In our study, we assumed that these parametrizations take place up to very big values of the scale factor $a$. This is a rather strong restriction. Therefore, the cases of variable $w$ different from the linear parametrizations require a separate investigation. For example, it was shown in [14] that exotic quark-gluon nuggets suitable to describe the dark sides of the Universe [21, 22], if they exist, can also be in agreement with the theory of scalar perturbations while being characterized by variable $w$.

\section{Acknowledgements}

Ö.A. acknowledges the support by TÜBİTAK Research Fellowship for Post-Doctoral Researchers (2218) and also the support from Koç University. The work of M.B.L. is supported by the Portuguese Agency "Fundação para a Ciência e Tecnologia" through an Investigador FCT Research contract, with reference IF/01442/2013/CP1196/CT0001. She also wishes to acknowledge the support from the Portuguese Grants PTDC/FIS/111032/2009 and PEstOE/MAT/UI0212/2014 and the partial support from the Basque government Grant No. IT592-13 (Spain). The work of M.E. is supported by NSF CREST award HRD-1345219 and NASA grant NNX09AV07A. A.Zh. acknowledges the hospitality of CENTRA/IST and UBI during the completion of a part of this work.

\section{Appendix: Adiabatic perturbations of perfect fluids with constant EoS pa- rameters}

In this appendix we obtain the fluctuations of the energy density for perfect fluids with a constant EoS parameter as a function of the scale factor $a$. For this purpose we consider the conservation equation (see, e.g., Eq. (2.74) in [20])

$$
\delta \varepsilon_{\mathrm{X}}^{\prime}+3 \frac{a^{\prime}}{a}\left(\delta \varepsilon_{\mathrm{X}}+\delta p_{\mathrm{X}}\right)+\left(\bar{\varepsilon}_{\mathrm{X}}+\bar{p}_{\mathrm{X}}\right)\left(\Delta v-3 \Phi^{\prime}\right)=0,
$$

where $\Delta$ is the Laplace operator with respect to the comoving coordinates and $v$ is the comoving peculiar velocity potential. We remark that we consider the fluctuations of perfect fluids arising due to inhomogeneities of dust-like matter (e.g., galaxies). These fluctuations also form their own inhomogeneities. Then the velocity potential $v$ characterizes dynamics of displacement of such inhomogeneities. For example, in the case of radiation, $v$ corresponds not to the speed of light but to the speed of displacement of photon concentrations. In the late Universe these displacement speeds are nonrelativistic and give much less contributions than $\Phi[10,12]$. Therefore, we drop the term containing $v$ in Eq. (A1). The gravitational potential is given by the formula (2.7): $\Phi=\varphi /\left(c^{2} a\right)$. We consider perfect fluids with a constant EoS parameter $w: \bar{p}_{\mathrm{X}}=w \bar{\varepsilon}_{\mathrm{X}}$. Hence, the conservation equation gives $\bar{\varepsilon}_{\mathrm{X}}=\bar{A} / a^{3(1+w)}$. For such 
fluids their adiabatic fluctuations also satisfy the same EoS: $\delta p_{\mathrm{X}}=w \delta \varepsilon_{\mathrm{X}}$. Then the general solution of Eq. (A1) may be written in the form

$$
\delta \varepsilon_{\mathrm{X}}=\frac{A}{a^{3(1+w)}}+\frac{3 \varphi(1+w) \bar{A}}{c^{2} a^{3(1+w)+1}},
$$

where $A$ is a constant of integration. This expression shows that in the case of the negative parameter $w<0$ both terms in the rhs satisfy the adopted accuracy because $3(1+w)+1<4$. However, the Einstein equations (in our case, in the form of Eqs. (2.17) and (2.18)) may impose additional restrictions on the form of $\delta \varepsilon_{\mathrm{X}}$. For example, the agreement with Eq. (2.22) where $\delta \varepsilon_{\operatorname{rad} 2}=0$ results in additional conditions: $A=0,-(1+w) / w=3(1+w)$. The latter condition holds true only for $w=-1$ and $w=-1 / 3$. Therefore,

$$
\delta \varepsilon_{-1 / 3}=\frac{2 \varphi}{c^{2}} \bar{A} \frac{1}{a^{3}}
$$

The conclusion that the value $w=-1 / 3$ is a particular one was also made from different reasoning in papers by Fulvio Melia (see, e.g., [23] and the references therein). It can be also easily seen that in the case $w=0$ the formula (A2) exactly reproduces Eq. (2.8) if we put $A=\delta \rho_{\mathrm{c}} c^{2}, \bar{A}=\bar{\rho}_{c} c^{2}$. In the case of radiation $w=1 / 3$ the second term in (A2) is of the order $O\left(1 / a^{5}\right)$ and should be dropped. Then, we get

$$
\delta \varepsilon_{\mathrm{rad}} \sim 1 / a^{4} .
$$

It is worth mentioning that in the general case of interacting fluids Eq. (A1) must contain their total energy densities and pressures. This case is beyond the scope of our paper where we concentrated exclusively on the standard perfect fluids without energy exchange between them.

There is also one more conservation equation (see, e.g., (2.75) in [20]). In our approach (that is when neglecting the peculiar velocities) this equation reads

$$
\sum_{i}\left[\delta p_{\mathrm{X}_{i}}+\left(\bar{\varepsilon}_{\mathrm{X}_{i}}+\bar{p}_{\mathrm{X}_{i}}\right) \Phi\right]=0 .
$$

Let us consider a model with four perfect fluids: dark energy represented by the cosmological constant, CDM, radiation and the $w=-1 / 3$ fluid. Then, (A5) takes the following form:

$$
\begin{aligned}
& \delta p_{\Lambda}+\left(\bar{\varepsilon}_{\Lambda}+\bar{p}_{\Lambda}\right) \Phi+\delta p_{\mathrm{CDM}}+\left(\bar{\varepsilon}_{\mathrm{CDM}}+\bar{p}_{\mathrm{CDM}}\right) \Phi \\
+ & \delta p_{\mathrm{rad}}+\left(\bar{\varepsilon}_{\mathrm{rad}}+\bar{p}_{\mathrm{rad}}\right) \Phi+\delta p_{-1 / 3}+\left(\bar{\varepsilon}_{-1 / 3}+\bar{p}_{-1 / 3}\right) \Phi=0 .
\end{aligned}
$$

Because $\delta p_{\Lambda}=0$ and $\bar{\varepsilon}_{\Lambda}=-\bar{p}_{\Lambda}$, the contribution from the $\Lambda$-term disappears. Now, taking into account that $\delta p_{\mathrm{CDM}}=\bar{p}_{\mathrm{CDM}}=0, \delta p_{\mathrm{rad}}=(1 / 3) \delta \varepsilon_{\mathrm{rad}}$ and $\left(\bar{\varepsilon}_{\mathrm{rad}}+\bar{p}_{\mathrm{rad}}\right) \Phi \sim O\left(1 / a^{5}\right)$, we get

$$
\delta \varepsilon_{\mathrm{rad}}=-3 \bar{\varepsilon}_{\mathrm{CDM}} \Phi=-\frac{3 \bar{\rho}_{\mathrm{c}} \varphi}{a^{4}}
$$

where $\bar{\varepsilon}_{\mathrm{CDM}}=\bar{\rho}_{\mathrm{C}} c^{2} / a^{3}$. This expression exactly coincides with the formula (2.10) in Sec. 2. Finally, keeping in mind that $\bar{p}_{-1 / 3}=-(1 / 3) \bar{\varepsilon}_{-1 / 3}$ and $\delta p_{-1 / 3}=-(1 / 3) \delta \varepsilon_{-1 / 3}$, we arrive at Eq. (A3). Therefore, we have shown the self-consistency of our approach. 


\section{References}

[1] P.A.R. Ade et al. [Planck Collaboration], Planck 2013 results. XVI. Cosmological parameters, Astron. Astrophys. 571 (2014) A16; arXiv:astro-ph/1303.5076; P.A.R. Ade et al. [Planck Collaboration], Planck 2015 results. XIII. Cosmological parameters; arXiv:astro-ph/1502.01589.

[2] A.D. Dolgov, Cosmic antigravity; arXiv:astro-ph/1206.3725.

[3] M. Chevallier and D. Polarski, Accelerating Universes with scaling dark matter, Int. J. Mod. Phys. D. 10 (2001) 213; arXiv:gr-qc/0009008.

[4] E.V. Linder, Exploring the expansion history of the Universe, Phys. Rev. Lett. 90 (2003) 091301; arXiv:astro-ph/0208512.

[5] V. Salzano, Y. Wang, I. Sendra and Ruth Lazkoz, Linear dark energy equation of state revealed by supernovae?, Mod. Phys. Lett. A 29 (2014) 1450008; arXiv:astro-ph/1211.1012.

[6] A.L. King, T.M. Davis, K. Denney, M. Vestergaard and D. Watson, High Redshift Standard Candles: Predicted cosmological constraints, MNRAS 441 (2014) 3454; arXiv:astro-ph/1311.2356.

[7] S. Capozziello, M. De Laurentis, O. Luongo and A.C. Ruggeri Cosmographic constraints and cosmic fluids, Galaxies 1(3) (2013) 216; arXiv:gr-qc/1312.1825.

[8] E. Aubourg et al. [BOSS Collaboration], Cosmological implications of baryon acoustic oscillation (BAO) measurements; arXiv:astro-ph/1411.1074.

[9] Ö. Akarsu, T. Dereli and J.A. Vazquez, A divergence-free parametrization for dynamical dark energy, JCAP 06 (2015) 049; arXiv:astro-ph/1501.07598.

[10] M. Eingorn and A. Zhuk, Hubble flows and gravitational potentials in observable Universe, JCAP 09 (2012) 026; arXiv:astro-ph/1205.2384.

[11] M. Eingorn, A. Kudinova and A. Zhuk, Dynamics of astrophysical objects against the cosmological background, JCAP 04 (2013) 010; arXiv:astro-ph/1211.4045.

[12] M. Eingorn and A. Zhuk, Remarks on mechanical approach to observable Universe, JCAP 05 (2014) 024; arXiv:astro-ph/1309.4924.

[13] A. Burgazli, M. Eingorn and A. Zhuk, Rigorous theoretical constraint on constant negative EoS parameter $w$ and its effect for the late Universe, Eur. Phys. J. C 75 (2015) 118; arXiv:astro-ph/1301.0418.

[14] M. Brilenkov, M. Eingorn, L. Jenkovszky and A. Zhuk, Scalar perturbations in cosmological models with quark nuggets, Eur. Phys. J. C 74 (2014) 3011; arXiv:astro-ph/1310.4540.

[15] M. Eingorn, J. Novák and A. Zhuk, $f(R)$ gravity: scalar perturbations in the late Universe, Eur. Phys. J. C 74 (2014) 3005; arXiv:astro-ph/1401.5410.

[16] A.R. Cooray and D. Huterer, Gravitational lensing as a probe of quintessence, Astrophys. J. 513 (1999) L95; arXiv:astro-ph/9901097.

[17] M. Eingorn and C. Kiefer, Scalar perturbations in cosmological models with dark energy - dark matter interaction, JCAP 07 (2015) 036; arXiv:gr-qc/1502.06912.

[18] L.D. Landau and E.M. Lifshitz, The Classical Theory of Fields, Fourth Edition: Volume 2 (Course of Theoretical Physics Series), Oxford Pergamon Press, Oxford (2000).

[19] V.F. Mukhanov, H.A. Feldman and R.H. Brandenberger, Theory of cosmological perturbations, Physics Reports 215 (1992) 203.

[20] D.S. Gorbunov and V.A. Rubakov, Introduction to the Theory of the Early Universe: Cosmological Perturbations and Inflationary Theory, World Scientific, Singapore (2011). 
[21] M. Brilenkov, M. Eingorn, L. Jenkovszky and A. Zhuk, Dark matter and dark energy from quark bag model, JCAP 08 (2013) 002; arXiv:astro-ph/1304.7521.

[22] A. Montiel, V. Salzano and R. Lazkoz, Observational constraints on the unified dark matter and dark energy model based on the quark bag model, Phys. Lett. B 733 (2014) 209; arXiv:astro-ph/1404.0388.

[23] F. Melia, The cosmic equation of state; arXiv:astro-ph/1411.5771. 